\title{
Recent Development in Applications of Optical MEMS: A Review
}

\author{
Poorvi K. Joshi \\ Assistant Professor, Department of Electronics Engineering, Shri Ramdeobaba College of Engineering and \\ Management, Ramdeo Tekadi, Gittikhadan, Katol Road, Nagpur 440013, India \\ Email:joshipk@rknec.edu
}

Received: $20^{\text {th }}$ September 2018, Accepted: $11^{\text {th }}$ October 2018, Published: $31^{\text {st }}$ October 2018

\begin{abstract}
Recent development of optical MEMS emphasis on application in the field of telecommunications, various sensors and micro mirror devices are few examples. Different optical devices and systems reported and explained the major difficulties in its development as well as performance. Advantages of optical MEMS are miniaturization, integration and mass production which create lot of potential to carry out research in this area. Focus of presented review is to explore the areas for future research work. Also, to identify the key and important issues related with optical MEMS and try to develop a technology to mitigate these issues.
\end{abstract}

\section{Keywords}

MEMS, MOEMS Devices, Sensors, Micro Mirrors

\section{Introduction}

Miniaturization of devices or mechanical elements is important issue in development of new technology. A micro-optoelctro-mechanical system (MOEMS) is miniaturized devices which measures and manipulates optical signals upon actuation of mechanical signals. Over a last decade it gains very importance for industrial application in terms of sensors or actuators. Recent advances in MOEMS technologies make optical system more robust and allow designing simpler. Now a days, applications of micro-electro-mechanical systems (MEMS) and MOEMS are in consumer, industrial, automotive, avionic, and biomedical fields. Various forces acting on devices, improved functionalities and environment conditions affecting the working of devices are the important issues in improvement of design and integration of MOEMS devices. The development in new technology paves way for wider applications of MOEMS devices for future. Packaging of optical component and placing of it on the mechanical structure are the key issue for precise working and functional reliability of the devices. MEMS scanning mirrors are the commonly used MOEMS devices and all have a movable mirror plate. It is very important to measure and control the displacement and/or rotation angle of the mirror plate. A miniaturized micromechanical element performs various optical functions; alter the profile or track intensity and phase of optical beam. All these actions are achieved by physical displacement like; revolution of mirrors, pressure, stresses, temperature variations which produces optical effect in MOEMS devices. Simulation technique based on beam tracing or by the scalar scattering theory where the scattering parameters are calibrated by comparison with experiential data. A widely used solver of Maxwell equations is based on the Rigorous Coupled-Wave Method (RCWA). In presented review applications of optical MEMS and issues related to optical MEMS devices applications are discussed.

\section{Recent Development in Optical MEMS}

MEMS optical sub-systems that are used within a optical communication system are optical fibers, lasers and laser diodes, optical receivers (photodetector), focusing lenses, diffraction lenses and gratings and mirrors. Advantages of optical MEMS are easy to manipulate light, simplified packaging, small size. Applications for MEMS have been developed in several fields: consumer products, gaming, aerospace, automotive, biomedical, chemical, optical displays, wireless and optical communications, fluidics. S. Saponara et.al [1] proposed 10-bit resistor-string digital-to-analog converter (DAC) for MOEMS micro mirror interfacing is realized in a $0.18-\mu \mathrm{m} \mathrm{BCD}$ technology. The key element is a folded resistor-string stage with a switch matrix and address decoders plus an output voltage buffer stage. They compared the proposed DAC to the state-of-the-art resistor-string converters in terms of bit resolution, linearity, and power consumption. The proposed DAC has the advantage of the smallest area occupation and Power-down circuitry is also implemented. M. Garrigues et.al [2] demonstrate the potential of micro-opto-electro-mechanical system (MOEMS) devices such as shutters, rotating mirrors based on III-V semiconductor materials, with special emphasis on applications for telecommunications. They demonstrated the use of MOEMS technology to enhance the capabilities of optical micro-cavity when strict confinement of the light is required in photonic devices, such as lasers. T. Niesel and A. Dietzel [3] implemented a micro-optical resonator gyroscope from which the necessity of micro alignment for two rotational axes becomes obvious. Micro-clamps allow aligned positioning and fixing of the double mirror elements in order to compensate initial rotational misalignments in the assembly of the resonator. They concluded that in future stiffer micro-spring structures will be investigated which 
can overcome all frictional forces during alignment. G. H. Dushaq et al [4] presented optically enabled inertia sensor with a dynamic range up to $10 \mathrm{~g}$ of operation. The TE and TM light modes in relatively low-index contrast Si3N4 suspended waveguide has been studied. In completely etched bottom cladding waveguide, TM mode is highly sensitive to any out of plane movement recording $\sim 25 \mathrm{~dB} / \mu \mathrm{m}$ change inlight intensity for $0.25 \mu \mathrm{m}$ Si3N4 width. H. Steiner et al. [5] suggested approach for measurement of static and angular displacement on inclined sensor using MOEMS transducer with non linear transfer characteristics. Two overlapping array, one array is deposited on fixed glass cover and other is etched on movable seismic mass of chip. Output from transducer is proportional to light flux modulated by these two aperture array. Static displacement resolution of $3.67 \mathrm{~nm}$ is measured and is compared with DC measurement approach which is $5.39 \mathrm{~nm}$. Also, the inclination sensors design shows improvement in resolution by factor of three. Frederic Zamkotsian et.al. [6] carried out cryogenic interferometric test to study the surface character of MOEMS deformable mirror under cryogenic and vacuum conditions. They designed cryogenic vacuum chamber 10-6 mbar and $160 \mathrm{~K}$ for Michelson interferometer to measure the performance of DM under harsh environment. They concluded that MOEMS DM from Iris adaptive optics has been tested successfully from ambient temperature to $160 \mathrm{~K}$. This study can be useful to achieve stability of device in harsh environment and improve operational stability.AleksandarJovic et.al. [7] proposed miniaturized single-chip integrated MOEMS scanner for large scanning range and all-in-one chip imaging system. They carried out system design, integration of different sub modules and lastly performance of MOEMS device. They found that lateral scanning range of $2000 \mu \mathrm{m}$ and $1000 \mu \mathrm{m}$ vertical is achieved with poor output of $170 \mathrm{~mW}$, within a area of $7 \times 10^{2} \mathrm{~mm}$. They suggested that this technology provides innovative solution for all-in-one chip imaging systems.Sundar Subramanian et. al. [8] proposes structure design of MOEMS accelerometer sensor for automobile passenger safety. Photonic crystal used for controlling mechanical device. Proposed structure shows light propagation and captivate in photonic crystal for displacement from 0 to 0.6 micrometer range. They found that wavelength shift of 0.001 for each micron displacement with Q factor of 6000 . They suggested that this technology can be used in form of MOEMS device in future for safety of passengers.A.Kainz et.al. [9] presents an analytical model for the air damping of laterally oscillating MEMS/MOEMS and surface forces responsible for air damping also studied. The shear forces also evaluated experimentally to quantify influence of hole. The developed model is tested with FVM (finite volume method) computations and simulation (Open FOAM). They found that model developed is having good agreement with computational model and measurement of MOEMS/MEMS test device. They suggested that this model can be used in MEMS devises used in ambient air and presented model can be checked for thermal noise.W. Hortschitza et.al [10] presented extremely sensitive low frequency (MOEMS) vibration sensor with low resonance frequency paves the way for micro-mechanical systems to be used in new fields of applications such as seismology or novel medical applications. Kainza et al [11] contributed towards highly integrated printable devices which will pave the way for future low-cost fabrication. They discussed the fringing problem of the OLED to achieve higher robustness and lifetime by improving the setup with an increasing level of integration and trying to change the OLED/OPD materials and technology. F. Ivaldi et al [12] implemented an innovative system for sensing big arrays of cantilevers with the use of one single laser source and a single small sized position sensitive detector. The implemented system is capable of performing high resolution and sensitivity measurements of resonance frequencies and deflection in the order of picometers. They compare their results with to a commercially available vibrometer and the experimental data have been found to be well in agreement. They proposed scope can be functionalization layers and their use as sensors with exceptional sensitivity. Alan Paterson et al [13] had achieved Simultaneous Q-switching and wavelength tuning of a solid-state laser for the first time through actuation of a single MOEMS mirror. A wavelength tuning range of $15.4 \mathrm{~nm}$ was achieved with laser pulse durations between $460 \mathrm{~ns}$ and $740 \mathrm{~ns}$ at a repetition rate of $2.06 \mathrm{kHz}$. M. Zanuccoli et al [14] implemented of RCWA to the calculation of optical reflectivity and to the geometry optimization of mirrors in MOEMS. These are critical components in terms of cost and performance. They adopted enhanced version of RCWA which allows to study the sensitivity of the optical properties to process variability and interfaces morphology. Russell Farrugia[15] done an in-depth analysis on the fluid-structure interactions of a micro-mirror resonating at high frequency and large scan angles. They discussed limitations of analytical formulations used to predict the damping moment of the mirror plate and vertical comb actuator. They have verified simulation results against measurements using a position sensitive detector. ShanshanGu-Stoppel et.al [16] developed micro mirrors operates on piezoelectric to attain large optical angle and high frequency. They designed and carried out FEM simulation for optimizing mirror design. They also investigated the accuracy of position sensing and close loop control of micro mirrors. They found that optical scan angle of $106^{\circ}$ and frequency of $45 \mathrm{kHz}$ at $22 \mathrm{~V}$ is achieved also measured Q-factor exceeding 5000 and power consumption of $10.3 \mathrm{~mW} . \mathrm{M}$ Balasubramanian et. al. [17] designed MOEMS accelerometer based on SOI grating coupler over a loaded diaphragm. These structures are resistant to corrosion, higher sensitivity, explosion and fire proof. They found that due to small size of coupler, constant displacement gradient throughout grating safely approximates. Uniform rise in period along grating with displacement gradient. The designed device has dynamic range of $7 \mathrm{~g}$, frequency $4.7 \mathrm{KHz}$, for sensitivity of detector -60 $\mathrm{dBm}$ and sensitivity of device is $25 \mu \mathrm{g}$. They suggested that accelerometer working in multiple directions and axis can be designed as this device is useful for unidirectional acceleration measurement. Also work can be extended to improve operating range of sensor. 


\section{Conclusion}

Focus of presented review is to explore the areas for future research work. Also, to identify the key and important issues related with optical MEMS devices and try to develop a technology to mitigate these issues. Following are the concluding points drawn from the literature review discussed in earlier section.

High sensitive low frequency (MOEMS) vibration sensor can be used for seismology and medical applications. Use of MOEMS deformable mirrors in harsh environment and to improve operational stability are key issues. There is scope of development of MEMS/MOEMS sensor analytical and simulation model for harsh/toxic environment, thermal variation or for critical applications. Integrated optics based on photonic crystals can be used to develop novel MOEMS devices. MOEMS accelerometer sensor with photonic crystal can be used for safety of passengers in future. MOEMS accelerometer sensor working in multiple directions and axis can be designed and to improve operating range of sensor. MOEMS vibrations sensors with higher sensitivity can be developed by adjusting the stiffness and mass (mechanical parameters) and also gain more bandwidth by decreasing the response frequency for displacement sensing. Improvement in MOEMS design and optical coating can cater to the applications such as range finding, target tracking and optical gas sensing. Development in the area of active materials for MOEMS devices combines the optical and micro mechanical functions. Integrating MEMS with organic components can be used for low cost fabrication of printable device. Reliability and sensitivity of MEMS/MOOEMS devices are technological issues to be taken care in future.

\section{References}

[1] S. Saponara, T. Baldetti and L. Fanucci, "A Cost-Effective 10-Bit D/A Converter for Digital-Input MOEMS Micro mirror Actuation", Hindawi Publishing Corporation VLSI Design, Volume 2010, Article ID 169079, pp: 1-7, doi:10.1155/2010/169079.

[2] M. Garrigues, J. L. Leclercq and P. Viktorovitch, "III-V Semiconductor based MOEMS devices for optical Telecommunications", Microelectronic Engineering, Volumes 61-62, July 2002, Pages 933-945,Doi: 10.1016/S01679317(02)00545-2.

[3] Thalke Niesel and Andreas Dietzel, "Micro-clamps for precise positioning of $120^{\circ}$ silicon double mirrors in a MOEMS gyroscope", Microelectronic Engineering, Volume 159, 15 June 2016, Pages 27-31, doi: 10.1016/j.mee.2016.02.029.

[4] G. H. Dushaq, T. Mulugeta and M. Rasras, "Micro-Opto-Mechanical Disk for Inertia Sensing", Photonic Sensors, March 2016, Volume 6, Issue 1, pp 78-84, doi: 10.1007/s13320-015-0294-4.

[5] H.Steiner, W.Hortschitz, A.Kainz, M.Stifter, A.Jachimowicz, J.Schalko, F.Keplinger, F.Kohl, "MOEMS transducer with a non-linear transfer characteristic for static displacement measurement applications on the example of an inclination sensor", Sensors and Actuators A: Physical, Volume 263, 15 August 2017, Pages 727-732, doi: 10.1016/j.sna.2017.05.035.

[6] Frederic Zamkotsian, Franck Marchis, Patrick Lanzoni, Rudy Barette, Michael Helmbrecht and Alex Teichman, "Operation of a MOEMS Deformable Mirror in Cryo: Challenges and Results", Micromachines2017, Vol. 8, 233; pp: 117, doi:10.3390/mi8080233.

[7]AleksandarJovic, Jose L. Rubio, Toshihiko Uto, KefeiHei, Juan Sancho, Nuria Sanchez, Kirill Zinoviev, Eduardo Margallo, Gregory Pandraud And Pasqualina M. Sarro, "A Highly Miniturized Single-Chip Moems Scanner For All-InOne Imaging Solution", Ieee Micro Electro Mechanical Systems (Mems), 21-25 Jan. 2018, Pp: 25-28, doi: 10.1109/Memsys.2018.8346472.

[8]Sundar Subramanian, Anup M. Upadhyaya and Preeta Sharan, "Structure Design of Photonic Crystal Based MOEMS Accelerometer Sensor for Supplemental Restraint System in Automobile Passenger Safety", Indian Journal of Science and Technology,Vol 10(29), August 2017, DOI: 10.17485/ijst/2017/v10i29/117327.

[9] A.Kainz, W. Hortschitz,H.Steiner, J.Schalko, A. Jachimowicz, F. Keplinger, "Accurate analytical model for air damping inlateral MEMS/MOEMS oscillators", Sensors and Actuators A: Physical, Volume 255, 1 March 2017, Pages 154-159, doi: 10.1016/j.sna.2016.12.023.

[10] W. Hortschitza, A. Kainzb, H. Steinera, M. Stiftera , F. Kohla, J. Schalkoa,b, T. Sautera , F. Keplingerba, "MOEMS Vibration Sensor for Advanced Low-Frequency Applications with pm Resolution", Procedia Engineering, Volume 87, 2014, Pages 835-838, doi: 10.1016/j.proeng.2014.11.282.

[11] A. Kainza, * , W. Hortschitzb , H. Steinerb , Yi-Hong Hongc , Chao-HsuanChend, Hsiao-Wen Zand , Hsin-FeiMenge , T. Sauterb , F. Keplingera, "MOEMS Vibration Sensor with Organic Semiconductor Readout", Procedia Engineering, Volume 168, 2016, Pages 1253-1256, doi: 10.1016/j.proeng.2016.11.439.

[12] F. Ivaldi, T. Bieniek, P. Janus, J. Zajac, P. Grabiec, W. Majstrzyk, D. Kopiec, T. Gotszalk, "New approach for a multi-cantilever arrays sensor system with advanced MOEMS readout", 46th European Solid-State Device Research Conference (ESSDERC), 12-15 Sept. 2016, doi: 10.1109/ESSDERC.2016.7599681. 
[13] A.Paterson, R. Bauer, W. Lubeigt, D. Uttamchandani, "Q-switched tunable solid-state laser using a MOEMS mirror", International Conference on Optical MEMS and Nanophotonics (OMN), 13-17 Aug. 2017, doi: 10.1109/OMN.2017.8051449.

[14] M. Zanuccoli, C. Fiegna, E. Cianci, C. Wiemer, A. Lamperti and G. Tallarida, L. Lamagna, S. Losa, S. Rossini, F. Vercesi, "Simulation of micro-mirrors for optical MEMS", International Conference on Simulation of Semiconductor Processes and Devices (SISPAD), 7-9 Sept. 2017, doi: 10.23919/SISPAD.2017.8085269.

[15] R. Farrugia, B. Portelli, I. Grech, D. Camilleri, O. Casha, J. Micallef, E. Gatt, "Air Damping Analysis in Resonating Micro-Mirrors", Symposium on Design, Test, Integration \& Packaging of MEMS and MOEMS (DTIP), 22-25 May 2018, doi: 10.1109/DTIP.2018.8394220.

[16] Dr. ShanshanGu-Stoppel, Thorsten Giese, Hans-Joachim Quenzer, Dr. Ulrich Hofmann und Prof. Wolfgang Benecke, "Piezoelectrically driven and sensed micro mirrors with large scan angles and precise closed-loop control", Proceeding, MikroSystemTechnikKongress, 23. - 25. Oktober 2017 in München. ISBN 978-3-8007-4491-6, pp: 203-206.

[17] M Balasubramanian, Shreyas Nandi, Shirin Fathima, Aparna S, Sai SrujanaVuppala, U Poornalakshmi, Prasant, Kumar Pattnaik, "MOEMS Accelerometer Based On Grating Coupler Integrated With Embossed Diaphragm", Conference on Lasers and Electro-Optics Pacific Rim (CLEO-PR), 31 july-4 aug. 2017, doi: 10.1109/cleopr.2017.8118814. 